\section{Selecting culprits in type 1 diabetes $\beta$-cell killing}

\author{
Åke Lernmark \\ University of Washington, R.H. Williams Laboratory, 1959 N.E. Pacific Street, Room K-165 HSC, Seattle, \\ Washington 98195, USA. Phone: (206) 543-5316; Fax: (206) 543-3169; E-mail: ake@u.washington.edu.
}

Type 1 diabetes is by several measures a remarkable disorder. Diabetes appears when $80-90 \%$ of the islet $\beta$-cells have been lost after being killed in a process strongly associated with autoimmune phenomena $(1,2)$. The rate of $\beta$-cell destruction before the clinical diagnosis of diabetes is made is not understood. Once the disease process has resulted in hyperglycemia that needs to be corrected with daily insulin injections, the point of no return has been reached; patients remain insulin-dependent for the rest of their lives. The disease may occur at any age and in individuals throughout the world.

The incidence rate is increasing steadily in the developed countries. Individuals from developing countries moving to Western societies often expe-

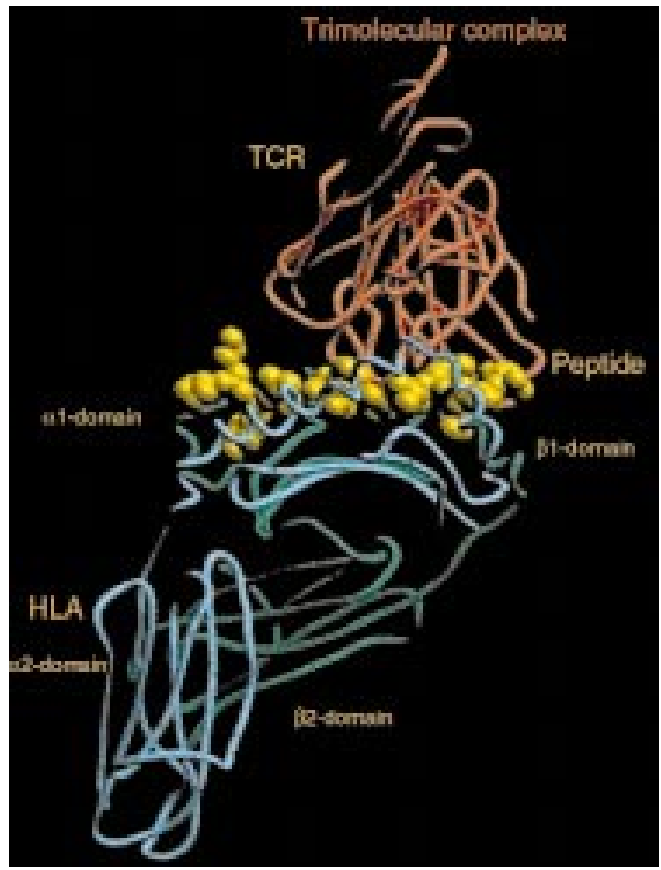

Figure 1

Model of HLA class II. The HLA class II molecule is composed of 2 polypeptide chains, $\alpha$ and $\beta$. The heterodimer forms a peptide-binding groove. The $\alpha 1$ domain, peptide, and $\beta 1$ domain is the trimolecular complex recognized by the T-cell receptor (TCR). The molecular model was generated and kindly provided by Peter Hjelmström (Department of Medicine, Karolinska Institute, Stockholm, Sweden). rience the disease, although they were unaware of it in their home countries. In countries with registries, incidence rates have increased to levels that border on epidemic (3). High-incidence regions such as Finland, Sardinia, and Sweden can expect that $1 \%$ of all newborn babies will develop type 1 diabetes sometime during their lifetime.

Considerable progress has been made in understanding the genetic etiology of this disease. Certain human leukocyte antigen (HLA) DQ and DR class II molecules are necessary but not sufficient for type 1 diabetes $(1,4)$. Two haplotypes, DQB1*0302-A1*0301 DRB1*04 and DQB1*0201-A1* 0501 DRB1*03 are most significant in this regard. Patients with type 1 diabetes from all over the world tend to have 1 or more copies of these haplotypes, and the highest risk for early age at onset is conferred by compound heterozygosity for each of these haplotypes (5, 6). The DQB1*0602 and DQB1*0603 alleles, on the other hand, are negatively associated with the disease, although their protective effect attenuates with age (5, 6). The varying risk with age suggests that the DQ and DR class II molecules play an active role, perhaps by presenting autoantigens that affect the pathogenetic process of $\beta$-cell killing.

Conformation-sensitive autoantibodies in diabetes pathogenesis. Whereas HLA is by far the most important factor in type 1 diabetes risk, immune abnormalities, in particular islet cell autoantibodies, are excellent pathogenetic markers. These autoantibodies are demonstrated in individuals who have been followed before the onset of diabetes, including firstdegree relatives (7) and individuals in the general population (8), as well as newborns (9), who have autoantibody mark- ers, often many years before the clinical diagnosis. In prospective family studies, it has been found that the presence of 2 or more of the autoantibodies to either glutamic acid decarboxylase (GAD65), insulinoma antigen 2 (IA-2), or insulin predicts type 1 diabetes (7). The rate of progression varies depending on the age of the subject, and many factors seem to affect the pathogenetic process leading to $\beta$-cell killing (10). Currently, the autoantibody markers are being used to identify at-risk individuals in trials to test whether insulin treatment can alter the immune response and prevent progression to clinical type 1 diabetes (11).

Because autoantibodies to GAD65, IA-2, and insulin are reliable predictors of ensuing clinical type 1 diabetes (12), it is thought that these autoantigens are also important to the cellular events that lead to the generation of autoantigen-specific CD4-positive T cells. The availability of such autoantigens, however, has not allowed reproducible identification of $\mathrm{T}$-cell responses, even in individuals who progress, as expected, to experience the disease. Whereas international workshops to standardize the measurement of islet cell antibodies have been highly successful (12), progress in developing reproducible $\mathrm{T}$ cell tests is still under way (13). The ability to measure reliable T-cell responses will be critical to our understanding of the role of autoantigen presentation and processing and the initiation and perseverance of a cell-mediated immune response in type 1 diabetes.

Several investigators have attempted to identify T-cell epitopes recognized by CD4-positive $T$ cells using overlapping synthetic peptides of both IA-2 (14) and GAD65 $(15,16)$. These analyses are complicated by the fact that peptides are presented by the class II molecules to T-cell receptors, which presumably recognize a linear epitope (Figure 1). Whereas the resulting activation of CD8-positive $\mathrm{T}$ cells that recognize HLA class I on islet $\beta$ cells remains speculative, the subsequent B-cell response is unambiguous and gen- 


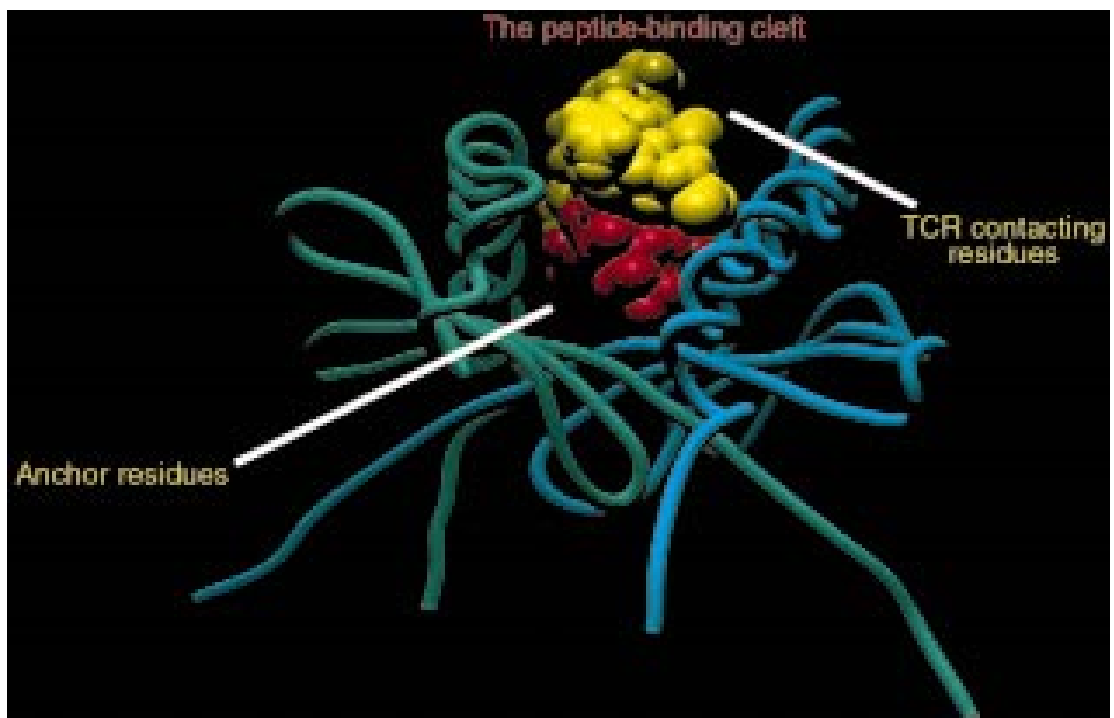

\section{Figure 2}

The peptide-binding cleft of the HLA class II molecule. The peptide is bound by virtue of the physicochemical interaction between the cleft and the anchor residues of the peptide. The TCR contacting residues along with residues on the $\alpha 1$ and $\beta 1$ domains (see Figure 1) determines $T$-cell recognition and would represent a NPPE. The molecular model was generated and kindly provided by Peter Hjelmström (for additional models, see http://depts.washington.edu/rhwlab/dq/intro.html).

erates autoantibodies that are uniquely conformation dependent. Autoantibody tests in solid-phase systems such as ELISA simply do not work (12). Only assays that maintain the conformation of IA-2, GAD65, and insulin detect autoantibodies with high diagnostic sensitivity and specificity (12). The features of autoantigen presentation to T-cell receptors on autoreactive CD4-positive T cells that lead to this unusual humoral response are not understood. Therefore, systems that allow the generation of naturally processed and presented epitopes (NPPEs) of a specific autoantigen have been needed badly.

Analyzing processed epitopes that drive autoantibody production. In the paper by Peakman and coworkers in the previous issue of the JCI, a lectin-based system was used to deliver the IA-2 autoantigen to immortalized lymphocytes that carry MHC class II molecules of the DRB $1 * 0401$ subtype - a common DR4 subtype that is strongly associated with type 1 diabetes allowing the authors to purify and study the associated NPPE (17). The selection of the intracellular portion of the IA-2 molecule was logical, because it is recognized by conformation-dependent autoantibodies (18). Individuals with IA-2 autoantibodies have predictably rapid progression to type 1 diabetes (19), especially if they also carry the DRB1*0401 allele (20). The trick Peakman et al. (17) developed to find the IA-2-derived peptide bound in the cleft built upon previous studies, in which B lymphocytes from individuals with unique HLA types were transformed with Epstein-Barr virus (EBV). Starting with large cultures of these lymphocytes, the class I or II molecules can be solubilized in detergent, and the peptide bound to the HLA can be eluted, purified by HPLC, and analyzed by microsequencing. Large databases are available to display the pattern of peptides that can bind to different HLA class I and class II molecules (http://bimas.dcrt.nih.gov/molbio/hla_bind). Using bulk EBV-transformed cells, however, has not allowed the identification of specific autoantigen peptides. Rather, sequences of known autoantigens have been conspicuously absent from these analyses. The present work applied high-resolution mass spectrometry to the analysis of NPPEs eluted from the immortalized lymphocytes, allowing Peakman et al. to identify several IA-2 peptides processed and presented by the IA-2loaded antigen-presented cells.

In analyzing the peptides bound to HLA-DR 0401, the authors demonstrate a limited number of specific peptides. The peptides seem to fulfill criteria of specific anchor residues to fit the
DRB $1 * 0401$ peptide-binding cleft (Figure 2). Importantly, testing the synthetic peptide in an in vitro proliferation test identified specific $T$-cell responses in type 1 diabetic patients who expressed DRB $1 * 0401$. As expected, not all type 1 diabetic patients responded to the peptide, nor was there a clear-cut relationship between $\mathrm{T}$-cell responses to the IA-2 peptide and the presence of IA2 autoantibodies. However, a much larger group of carefully selected patients needs to be investigated. In none of 8 nondiabetic DRB1*0401-positive individuals did peripheral blood $\mathrm{T}$ cells respond to any of the 5 IA- 2 peptides identified. It remains unclear whether the processing and presentation of any one of the peptides would suffice to initiate IA-2 autoreactivity, or whether multiple epitopes must be recognized to promote disease progression. The T cells were from patients with already established type 1 diabetes, so significant epitope-spreading might have taken place before clinical diagnosis. Type 1 diabetes patients may have islet cell autoantibodies for several years before the clinical diagnosis. The conformationdependent IA-2 autoantibodies in type 1 diabetes bind to domains containing these peptides $(18,21)$, and it will now be possible to test the hypothesis that $\mathrm{T}$ cell autoreactivity is related to epitopespecific autoantibodies.

This study clearly represents an important step toward understanding the mechanism by which autoantigens are processed and presented. However, antigen-presenting cells may be defective in type 1 diabetes (22), and differences in processing may result in the presentation of distinct set of peptides in different patients (23). This experimental approach therefore needs to be individualized to permit the study of multiple pathways that lead to antigen processing and presentation with HLADRB1*0401. The present study focuses on epitope presentation to $\mathrm{T}$ cells, rather than downstream development of specific conformation-dependent autoantibodies, and its success should encourage further investigations on the mechanism of HLA selection of autoreactive peptides. In this regard, it is plausible that $\mathrm{T}$ cells that respond to continuous epitopes from self proteins are clonally deleted early in immune development; further studies will be needed to characterize the epitopes and cellular interactions that promote conformation- 
dependent auto-immunity. In addition, if the IA-2 peptide T-cell study is reproduced by other groups using larger sets of patients and controls, it could provide the basis of standardized T-cell assays.

The study by Peakman et al. (17) should also encourage the development of novel reagents to block T-cell responses. It is conceivable that by identifying the specific anchoring residues, the contact residues to the T-cell receptor (see Figure 2) may be modified to prevent T-cell receptor recognition. Modified peptides may be tested to determine if they block or compete with the endogenously loaded peptides. Many investigators have advocated the use of peptides to interfere with antigen presentation and block Tcell proliferation in type 1 diabetes. The present approach of selecting T-cell epitopes should allow studies of peptides that either stimulate or inhibit in vitro Tcell proliferation. This work may represent an important step toward future clinical trials using peptides either to induce tolerance to specific autoantigens in type 1 diabetes or to block $\beta$-cell killing. In light of the worldwide increased incidence of childhood type 1 diabetes, such tools are needed urgently.

1. Schranz, D., and Lernmark, Å. 1998. Immunology in diabetes: an update. Diabetes Metab. Rev. 14:3-29.

2. Bach, J.-F. 1994. Insulin-dependent diabetes mellitus as an autoimmune disease. Endocr. Rev. 15:516-542.
3. Green, A., Gale, E.A., and Patterson, C.C. 1992. Incidence of childhood-onset insulin-dependent diabetes mellitus: the EURODIAB ACE Study. Lancet. 339:905-909.

4. Nepom, G.T., and Kwok, W.W. 1998. Molecular basis for HLA-DQ associations with IDDM. Diabetes. 47:1177-1184

5. Graham, J., et al. 1999. Negative association between type 1 diabetes and HLA DQB1*0602DQA1*0102 is attenuated with age at onset. Swedish Childhood Diabetes Study Group. Eur. J. Immunogenet. 26:117-127.

6. Caillat-Zucman, A., et al. 1992. Age-dependent HLA genetic heterogeneity of type 1 insulin-dependent diabetes mellitus. J. Clin. Invest. 90:2242-2250.

7. Verge, C.F., et al. 1996. Prediction of type I diabetes in first-degree relatives using a combination of insulin, GAD, and ICA512bdc/IA-2 autoantibodies. Diabetes. 45:926-933.

8. Bingley, P.J., et al. 1997. Prediction of IDDM in the general population: strategies based on combinations of autoantibody markers. Diabetes. 46:1701-1710.

9. Lindberg, B., et al. 1999. Islet autoantibodies in cord blood from children who developed type 1 diabetes before 15 years of age. Diabetologia. In press.

10. Greenbaum, C.J., Sears, K.L., Kahn, S.E., and Palmer,J.P. 1999. Relationship of beta-cell function and autoantibodies to progression and nonprogression of subclinical type 1 diabetes: follow-up of the Seattle Family Study. Diabetes. 48:170-175.

11. Group, D.-S. 1995. The Diabetes Prevention Trial-type 1 diabetes (DPT-1): implementation of screening and staging of relatives. Transplant Proc. 27:3377.

12. Verge, C.F., et al. 1998. Combined use of autoantibodies (IA-2) autoantibody, GAD autoantibody, insulin autoantibody, cytoplasmic islet cell antibodies in type 1 diabetes: Combinatorial Islet Autoantibody Workshop. Diabetes. 47:1857-1866.

13. Roep, B.O., et al. 1999. Autoreactive T cell responses in insulin-dependent (type 1) diabetes mellitus. Report of the First International Workshop for Standardization of $\mathrm{T}$ cell assays. $J$. Autoimmun. 13:267-282.

14. Harfouch-Hammoud, E., et al. 1999. Identifica- tion of peptides from autoantigens GAD65 and IA-2 that bind to HLA class II molecules predisposing to or protecting from type 1 diabetes. Diabetes. 48:1937-1947.

15. Wicker, L.S., et al. 1996. Naturally processed T cell epitopes from human glutamic acid decarboxylase identified using mice transgenic for the type 1 diabetes-associated human MHC class II allele, DRB1*0401. J. Clin. Invest. 98:2597-2603.

16. Endl, J., et al. 1997. Identification of naturally processed $\mathrm{T}$ cell epitopes from glutamic acid decarboxylase presented in the context of HLA$\mathrm{DR}$ alleles by $\mathrm{T}$ lymphocytes of recent onset IDDM patients. J. Clin. Invest. 99:2405-2415.

17. Peakman, M., et al. 1999. Naturally processed and presented epitopes of the islet cell autoantigen IA-2 eluted from HLA-DR4.J. Clin. Invest. 104:1449-1457

18. Lampasona, V., et al. 1996. Autoantibodies in insulin-dependent diabetes recognize distinct cytoplasmic domains of the protein tyrosine phosphatase-like IA-2 autoantigen. J. Immunol. 157:2707-2711.

19. Christie, M.R., et al. 1994. Antibodies to islet 37k antigen, but not to glutamate decarboxylase, discriminate rapid progression to IDDM in endocrine autoimmunity. Diabetes. 43:1254-1259.

20. Sanjeevi, C.B., et al. 1998. Association between autoantibody markers and subtypes of DR4 and DR4-DQ in Swedish children with insulindependent diabetes reveals closer association of tyrosine pyrophosphatase autoimmunity with DR4 than DQ8. Tissue Antigens. 51:281-286.

21. Xie, H., et al. 1997. Autoantibodies to IA-2 and IA2 beta in insulin-dependent diabetes mellitus recognize conformational epitopes: location of the $37-$ and $40-\mathrm{kDa}$ fragments determined. J. Immunol. 159:3662-3667.

22. Litherland, S.A., et al. 1999. Aberrant prostaglandin synthase 2 expression defines an antigen-presenting cell defect for insulin-dependent diabetes mellitus. J. Clin. Invest. 104:515-523.

23. Reijonen, H., Elliott, J.F., van Endert, P., and Nepom, G. 1999. Differential presentation of glutamic acid decarboxylase 65 (GAD65) T cell epitopes among HLA-DRB1*0401-positive individuals. J. Immunol. 163:1674-1681. 